\title{
CD1d highly expressed on DCs reduces lung tumor burden by enhancing antitumor immunity
}

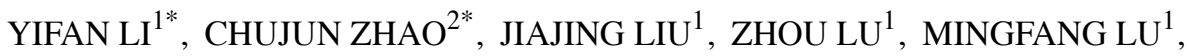 \\ JIE $\mathrm{GU}^{3}$ and RONGHUA LIU ${ }^{1,4}$ \\ ${ }^{1}$ Department of Immunology, School of Basic Medical Sciences, and Key Laboratory of \\ Medical Epigenetics and Metabolism, Institutes of Biomedical Sciences, Fudan University, Shanghai 200032, \\ P.R. China; ${ }^{2}$ Northfield Mount Hermon School, Northfield, MA 01354, USA; ${ }^{3}$ Department of Thoracic Surgery, \\ The Affiliated Zhongshan Hospital of Fudan University; ${ }^{4}$ Department of General Surgery, \\ Shanghai Fifth People's Hospital, Fudan University, Shanghai 200032, P.R. China
}

Received October 29, 2018; Accepted February 21, 2019

DOI: 10.3892 /or.2019.7037

\begin{abstract}
Dendritic cells (DCs), as professional antigenpresenting cells are essential for the initial activation of adaptive antitumor immunity. CD1d is considered to present phospholipid and glycosphingolipid antigens to NKT cells. However, it is currently unknown whether CD1d expression on DCs is capable of enhancing antitumor immunity, particularly T-cell related immunity. We observed that CD1d was predominantly expressed on DCs in 3LL tumor-bearing mice, whilst a deficiency of CD1d promoted tumor growth. Notably, CD1d expression on DCs was not only required for presenting antigen to NKT cells, but also markedly promoted $\mathrm{CD}^{+} \mathrm{T}$ and $\mathrm{CD}^{+} \mathrm{T}$ cell activation, particularly cytotoxic $\mathrm{T}$ cells. All the $\mathrm{T}$ cells (NKT, CD4 ${ }^{+} \mathrm{T}$ and $\mathrm{CD} 8^{+} \mathrm{T}$ cells) upregulated CD69, CD107a and IFN- $\gamma$ after the adoptive transfer of CD1d-positive DCs (CD1d ${ }^{+}$DCs) and tumor growth
\end{abstract}

Correspondence to: Professor Ronghua Liu, Department of Immunology, School of Basic Medical Sciences, and Key Laboratory of Medical Epigenetics and Metabolism, Institutes of Biomedical Sciences, Fudan University, 138 Yi Xueyuan Road, Shanghai 200032, P.R. China

E-mail: ronghualiu@fudan.edu.cn

Dr Jie Gu, Department of Thoracic Surgery, The Affiliated Zhongshan Hospital of Fudan University, 180 Fenglin Road, Shanghai 200032, P.R. China

E-mail: gu.jie3@zs-hospital.sh.cn

*Contributed equally

Abbreviations: DCs, dendritic cells; APCs, antigen-presenting cells; NKT, natural killer T; $\alpha$-GalCer, $\alpha$-galactosylceramide; DT, diphtheria toxin; FACS, fluorescence-activated cell sorter; Ag, antigen; TAAs, tumor-associated antigens; GZMB, granzyme B

Key words: CD1d, tumor, natural killer $\mathrm{T}$ cell, $\mathrm{T}$ cells, dendritic cells was suppressed. With regard to the mechanism, we revealed that $\mathrm{CD} 1 \mathrm{~d}^{+} \mathrm{DCs}$ were concomitant with a higher expression of costimulatory molecules (CD40, CD80 and CD86) and $\mathrm{MHCI} / \mathrm{II}$, which are essential for DCs to present antigens to $\mathrm{T}$ cells. Consistently, CD1d ${ }^{+} \mathrm{DCs}$ displayed stronger activation-associated-ERK $1 / 2$ and $\mathrm{NF}-\kappa \mathrm{B}$ signals; whereas JAK2-STAT3/6 signaling was required for maintaining a high level of CD1d on DCs. In lung cancer patients, the antitumor activities of all the $\mathrm{T}$ cells were enhanced with the increase of CD1d ${ }^{+}$DCs. Analysis of TCGA data revealed that high levels of CD1d indicated better outcomes for patients. Collectively, CD1d enhanced DC-based antitumor immunity, not only by targeting NKT, but also by activating $\mathrm{CD}^{+}{ }^{+} \mathrm{T}$ and $\mathrm{CD}^{+} \mathrm{T}$ cells. CD1d ${ }^{+} \mathrm{DCs}$ may be superior to the bulk population of DCs in cancer immunotherapy.

\section{Introduction}

Dendritic cells (DCs) are highly specialized professional antigen-presenting cells (APCs) that link antigen-independent innate immunity and antigen-specific adaptive immunity, essential for the initial activation of antitumor immunity $(1,2)$. As the first successful cancer vaccine, a DC vaccine named Provenge (which treats prostate carcinoma) was approved by the FDA in 2010, and successfully prolonged life for four months, implying the potential clinical significance of DCs in tumor immunotherapy $(3,4)$. The effective activation of antitumor immune responses strongly depends on efficient tumor antigen presentation from DCs (5). In this process, the expression of MHC-I/II and costimulatory molecules (such as CD80 and CD86) on DCs is required (6). This is necessary at the priming phase of antitumor immunity, particularly for $\mathrm{T}$ cell-dependent immunity. Therefore, widely improving these molecules to boost antigen-presenting function will be helpful for enhancing DC-based antitumor immunity.

CD1d is an alternative, MHC class I-like glycoprotein (7). The most extensively described, CD1d can bind and present antigens such as lipid and glycolipids, particularly to natural killer T (NKT) cells. These antigens can be both endogenous and exogenous, from invaded pathogens, or endogenous, 
synthesized by activated APCs (8-10). CD1d has been declared as a regulator in immune disorders such as infection, autoimmune and inflammatory diseases. By targeting NKT cells, CD1d has been revealed to function as a suppressor in tumors (11-15). Although CDld can be expressed by cells of both myeloid (monocytes and macrophages) and lymphoid lineages $(16,17)$, as well as non-hematopoietic cells (e.g., epithelial and vascular smooth muscle cells), it is still unclear which cells are the major source of CDld in tumors. Moreover, there is little direct evidence that CD1d is a potential target for enhancing antitumor effects of $\mathrm{CD} 4^{+} \mathrm{T}$ and $\mathrm{CD} 8^{+} \mathrm{T}$ cells, not only for NKT cells.

In the present study, by comparing CD1d expression on different APCs in 3LL tumor-bearing mice, we found that DCs had the highest expression of CD1d. Lung cancer patients with more CD1d-positive DCs (CD1d $\left.{ }^{+} \mathrm{DCs}\right)$ had an enhanced activity of both NKT, CD4 ${ }^{+} \mathrm{T}$ and $\mathrm{CD} 8^{+} \mathrm{T}$ cells. CD1d $\mathrm{d}^{+} \mathrm{DCs}$ highly expressed MHCs and costimulatory molecules such as CD80, CD86 and CD40, displaying a stronger ability in presenting antigens to $\mathrm{T}$ cells. The adoptive transfer of CD1d ${ }^{+}$DCs activated not only NKT but also $C D 4^{+} T$ and $\mathrm{CD} 8^{+} \mathrm{T}$ cells, subsequently suppressing tumor growth. The activation of immune responses by CD1d may provide better clinical outcomes in lung cancer patients.

\section{Materials and methods}

Clinical samples. The adjacent normal and neoplastic tissues were obtained from nine lung cancer patients ( 2 females and 7 males), (female, 39 years old; male, 64 years old; male, 64 years old; male, 66 years old; female, 39 years old; male, 69 years old; male, 37 years old; male, 61 years old; and male, 65 years old; the mean age of the patients was 56 years) who underwent complete surgical resections at Zhongshan Hospital (Shanghai, China). Informed consents for the use of the samples were obtained from all patients. The study was approved by the Zhongshan Hospital Research Ethics Committee (Shanghai, China).

Clinical sample preparation. Clinical adjacent normal and neoplastic tissues were collected in RPMI-1640 medium (Sigma-Aldrich; Merck KGaA, Darmstadt, Germany). The tissues were cut into small pieces with scissors. They were then digested with $0.5 \mathrm{mg} \mathrm{ml}^{-1}$ collagenase $\mathrm{D}$ and $0.1 \mathrm{mg} \mathrm{ml}^{-1}$ DNase I (Roche Applied Science, Mannheim, Germany) for $60 \mathrm{~min}$ at $37^{\circ} \mathrm{C}$ to isolate lymphocytes. For lymphocyte preparation, remaining tissues were mashed through a $70-\mu \mathrm{m}$ cell strainer (BD Biosciences, Franklin Lakes, NJ, USA) before FACS detection.

Mice and reagent. Forty-five $\mathrm{CD} 1 \mathrm{~d}$ gene knockout (KO) mice $(\mathrm{B} 6(\mathrm{C})-\mathrm{CD} 1 \mathrm{~d}<\mathrm{tm} 1.2 \mathrm{Aben}>/ \mathrm{J})$ were obtained from The Jackson Laboratory (Bar Harbor, ME, USA) and were bred in specific pathogen-free conditions $\left(18-29^{\circ} \mathrm{C}\right.$; the daily temperature difference was below than $3^{\circ} \mathrm{C}$; the humidity was $40-70 \%$; the air velocity was $<0.18 \mathrm{~m} / \mathrm{s}$; the gradient of room air pressure difference was 20-50 Pa) at the Department of Laboratory Animal Science, Fudan University (Shanghai, China). Eighty-six C57BL/6 mice from the Shanghai SLAC Laboratory Animal Co., Ltd. (Shanghai, China) were used for all experiments. Twenty-eight CD11C-DTR mice were kindly donated by Professor Zhinan Yin (College of Life Sciences, Nankai University, Tianjin, China). All the mice were female and their weight was $20 \mathrm{~g}$. All experiments were performed under protocols approved by the Ethics Committee of Animal Care and Use, Department of Laboratory Animal Science, at Fudan University (Shanghai, China; approval no. 201901002Z). Diphtheria toxin receptor (DTR)-mediated conditional cell ablation in mice is a widely used system of limitations conditional on cell depletion strategies. As previously reported, injection of DT can deplete DCs in vivo (18). In the present study, the CD11c.DTR mouse was selected to deplete DCs, and then characterize the role of CDld ${ }^{+} \mathrm{DCs}$ and CD1d-KO DCs in a 3LL tumor-bearing model. All mice used were aged 6-12 weeks. All animal experiments complied with the National Institute of Health's Guide for the Care and Use of Laboratory Animals (NIH Publications no. 8023, revised in 1978). The $\alpha$-GalCer used in the present study was donated by Abcam (Cambridge, UK).

TCGA data analysis. The Kaplan-Meier plots summarized correlations between the mRNA expression level of CD1d and lung cancer patient survival and tumor stage, which are based on the TCGA data from the Human Protein Atlas (https://www.proteinatlas.org/). Patients were divided into one of two groups 'low $(n=723)$ ' or 'high $(n=271)$ ' based on the level of CD1d. The cut-off was equal to 1.7 fragments/kilobase million (FPKM). Based on a standard score (z-score), combining the relative levels of NKT cell markers (CD161 and $C D 3)$, $\mathrm{T}$ cell markers $(C D 3$ or $C D 8)$ and antitumor response-related genes [CD161, CD3, CD69, IFN- $\gamma, G Z M B$ (granzyme $B$ ) and $C D 107 a$ ], a score of activated NKT, CD4 ${ }^{+} \mathrm{T}$ and $\mathrm{CD} 8^{+} \mathrm{T}$ cells was respectively calculated. The scores of MHC-I/II and costimulatory molecules were calculated based on the related genes $(H L A-A / B / C, H L A-D M A / D O A / D Q A 1$, $H L A-D R A, C D 80, C D 86$ and $C D 40)$.

Tumor-bearing mouse model. 3LL tumor cells (an undifferentiated lung cancer cell line) were injected into wild-type (WT) and CD1d-KO mice. Briefly, 3LL cells were cultured in Dulbecco's modified Eagle's medium (DMEM; Welgene, Inc., Daegu, Korea) supplemented with $10 \%$ fetal bovine serum (FBS; Wisent Bioproducts, St. Bruno, QC, Canada). The cultured cells were suspended in phosphate-buffered saline (PBS) and $5 \times 10^{5}$ cells/mouse (suspended in $0.1 \mathrm{ml}$ PBS) were then injected into the upper flank of WT and CDld-KO mice. In addition, C57BL/6 mice without tumor cell inoculation were treated with corn oil alone as the normal control group. Tumor volumes were assessed once every three days. On day 28 , the mice were euthanized by intraperitoneal injection of overdose pentobarbital sodium $(200 \mathrm{mg} / \mathrm{kg})$. The tumor tissue, spleen, draining lymph node (LN), and body weights were measured and observed (19).

Flow cytometric analysis. Flow cytometric data were acquired on a fluorescence-activated cell sorter (FACSCalibur; BD Biosciences) and data were analyzed with FlowJo 10.0.7 software (Tree Star, Inc., Ashland, OR, USA). Single-cell suspensions were prepared from the tumor tissues, draining LNs and spleens of WT and CD1d-KO mice after tumor 
inoculation. The cells were resuspended in PBS with antibodies (Abs) conjugated with FITC, PE, BV421 or allophycocyanin, which were against CD45 (dilution 1:200; cat. no. MCD4528; BD Biosciences), CD11C (dilution 1:200; cat. no. 11-0114-82; eBioscience; Thermo Fisher Scientific, Inc., Waltham, MA), NK1.1 (dilution 1:200; cat. no. 12-5941; eBioscience; Thermo Fisher Scientific, Inc.), CD69 (dilution 1:200; cat. no. 553236; BD Biosciences), CD80 (dilution 1:200; cat. no. 11-0801; eBioscience; Thermo Fisher Scientific, Inc.), CD86 (dilution 1:200; cat. no. 48-0862; eBioscience; Thermo Fisher Scientific, Inc.), MHC-I (dilution 1:100; cat. no. 48-5958; eBioscience; Thermo Fisher Scientific, Inc.), MHC-II (dilution 1:200; cat. no. 12-5320; eBioscience; Thermo Fisher Scientific, Inc.), CD19 (1:200; cat. no. 48-0193; eBioscience; Thermo Fisher Scientific, Inc.), CD3 (dilution 1:200; cat. no. 12-0031; eBioscience; Thermo Fisher Scientific, Inc.), CD40 (dilution 1:200; cat. no. 12-0401; eBioscience; Thermo Fisher Scientific, Inc.), CD107a (dilution 1:200; cat. no. 53-1071; eBioscience; Thermo Fisher Scientific, Inc.), CD1d (dilution 1:200; cat. no. 12-2019; eBioscience; Thermo Fisher Scientific, Inc.), CD8 (dilution 1:200; cat. no. 100711; BioLegend, San Jose, CA, USA), CD11b (dilution 1:200; cat. no. 47-0112; eBioscience; Thermo Fisher Scientific, Inc.), IFN- $\gamma$ (dilution 1:100; cat. no. 12-7311; eBioscience; Thermo Fisher Scientific, Inc.), pSTAT6 (dilution 1:100; cat. no. 12-9013-42; eBioscience; Thermo Fisher Scientific, Inc.) pSTAT3 (dilution 1:200; cat. no. 12-9033-42; eBioscience; Thermo Fisher Scientific, Inc.) and MHC-I, HLA-A/B/C (dilution 1:200; cat. no. 311410; BioLegend). Proper isotype Abs were used as the control. For intracellular detection, Cell Stimulation Cocktail (eBioscience; Thermo Fisher Scientific, Inc.) was used for $5 \mathrm{~h}$ at $37^{\circ} \mathrm{C}$ before FACS analysis. After stimulation, the cells were stained using antibodies against surface markers CD45, CD11C, NK1.1 and CD1d and then treated with Intracellular Fixation and Permeabilization buffer (eBioscience; Thermo Fisher Scientific, Inc.) (20).

Isolation of DC cells. DCs isolated from naive C57BL/6 WT mice splenocytes were purified by negative selection using the EasySep ${ }^{\mathrm{TM}}$ Mouse DC Cell Isolation kit (Stemcell Technologies, Inc., Vancouver, BC, Canada). The purity was $>70 \%$ as determined by FACS. Primary DCs were then cultured in RPMI-1640 medium (Gibco; Thermo Fisher Scientific, Inc.) with $10 \%$ FBS (Gibco; Thermo Fisher Scientific, Inc.), $50 \mathrm{mM} \beta$-mercaptoethanol (Sigma-Aldrich; Merck KGaA) and $1 \mathrm{X}$ antibiotic mixture (Gibco; Thermo Fisher Scientific, Inc.) at $37^{\circ} \mathrm{C}$ in 96-well plates and stimulated with GM-CSF $(20 \mathrm{ng} / \mathrm{ml}$; Sigma-Aldrich; Merck KGaA). To isolate CDld ${ }^{+}$DCs and the paired CD1dDCs, single cells from tumor-bearing mice were labeled with the cell surface markers for CD1d ${ }^{+} \mathrm{DCs}$, defined as $\mathrm{CD}_{4}{ }^{+} \mathrm{CD} 11 \mathrm{C}^{+}$and $\mathrm{CD} \mathrm{d}^{+}$. They were sorted by FACS (MoFlo; Beckman Coulter, Inc., Brea, CA, USA).

Adoptive transfer. For systemic DC depletion, CD11C-DTR transgenic mice were injected intraperitoneally with $4 \mathrm{ng} / \mathrm{g}$ body weight diphtheria toxin (DT; in PBS; Sigma-Aldrich; Merck KGaA; product no. D-0564, formerly D-2918). After $48 \mathrm{~h}$, they were treated with CD1d ${ }^{+}$DCs and CD1d-KO DCs and injected with 3LL tumor cells as aforementioned. The DT-DC cycle was performed for another four rounds per week and the mice were euthanized on day 28 of tumor transplantation.

DCs stimulate NKT cells in vitro. Splenic DCs $\left(2 \times 10^{5}\right)$ from WT or CD1d-KO mice were stimulated by the injection of $\alpha$-GalCer $(250 \mathrm{ng} / \mathrm{ml})$ and GM-CSF $(20 \mathrm{ng} / \mathrm{ml})$ in 96-well U-bottomed plates (Corning Costar Co., Ltd., Tokyo, Japan) overnight, and then co-cultured with purified NKT cells $\left(2 \times 10^{5}\right)$ from WT mice. NKT cells were sorted with MoFlo High-Performance Cell Sorter (Beckman Coulter, Inc.). After incubation for $36 \mathrm{~h}$, the culture supernatants were harvested to detect cytokine levels by FACS.

Cell treatment and western blot analysis. For the quantitative detection of signals in CD1d ${ }^{+}$DCs, DCs of tumor-bearing mice were marked using CD1d and CD11C, then CD1d ${ }^{+}$DCs and the paired CD1d-DCs were isolated by FACS. CD1d ${ }^{+} D C$ cells were treated with pSTAT3 inhibitor Pyridone 6 (10 nM; Beyotime Institute of Biotechnology, Shanghai, China) and pSTAT6 inhibitor AS1517499 (10 nM; Axon Medchem BV, Groningen, The Netherlands), and then the expression levels of CD1d were detected by FACS. Furthermore, the total protein was extracted after cell lysis and immunoblotting analysis was performed, as previously reported (21). The protein was probed with the primary antibodies overnight at $4{ }^{\circ} \mathrm{C}$, then incubated with a anti-rabbit secondary antibody conjugated with horseradish peroxidase (HRP) (dilution 1:5,000; cat. no. HRP-7077; Cell Signaling Technology, Inc., Danvers, MA, USA). Enhanced chemiluminescence (cat. no. 34095; Thermo Fisher Scientific, Inc.) was used for detection (22). The primary antibodies were specific for phosphorylated ERK1/2 (dilution 1:1,000; cat. no. 4370; Cell Signaling Technology, Inc.), p-p65 (dilution 1:1,000; cat. no. 3033; Cell Signaling Technology, Inc.) and pJAK2 (dilution 1:1,000; cat. no. 3771; Cell Signaling Technology, Inc.). $\beta$-actin was used as an internal reference and detected using HRP-conjugated anti-mouse $\beta$-actin (dilution 1:5,000; cat. no. HRP-60008; ProteinTech Group, Inc., Chicago, IL, USA).

Statistical analysis. Data were analyzed using the GraphPad Prism software (version 6; GraphPad Software Inc., La Jolla, CA, USA) and were presented as the means \pm standard error of the mean (SEM). Student's unpaired t-test or unpaired t-test with Welch's correction were used to analyze intergroup differences for two groups. One-way analysis of variance (ANOVA) and the Tukey's multiple comparisons test was used to analyze more than two groups. Pearson's correlation coefficient was used to analyze the correlation between groups. $\mathrm{P}<0.05$ was considered to indicate a statistically significant difference.

\section{Results}

The expression of CDId is correlated with extended survival of lung cancer patients, indicating stronger antitumor immunity. To investigate the potential significance of CD1d in tumors, we analyzed CD1d expression in 994 human lung cancer samples from databases of The Cancer Genome Atlas (TCGA) in the Human Protein Atlas. Data revealed that the expression of CD1d was positively correlated with the overall 


\section{A} CD1d in lung cancer
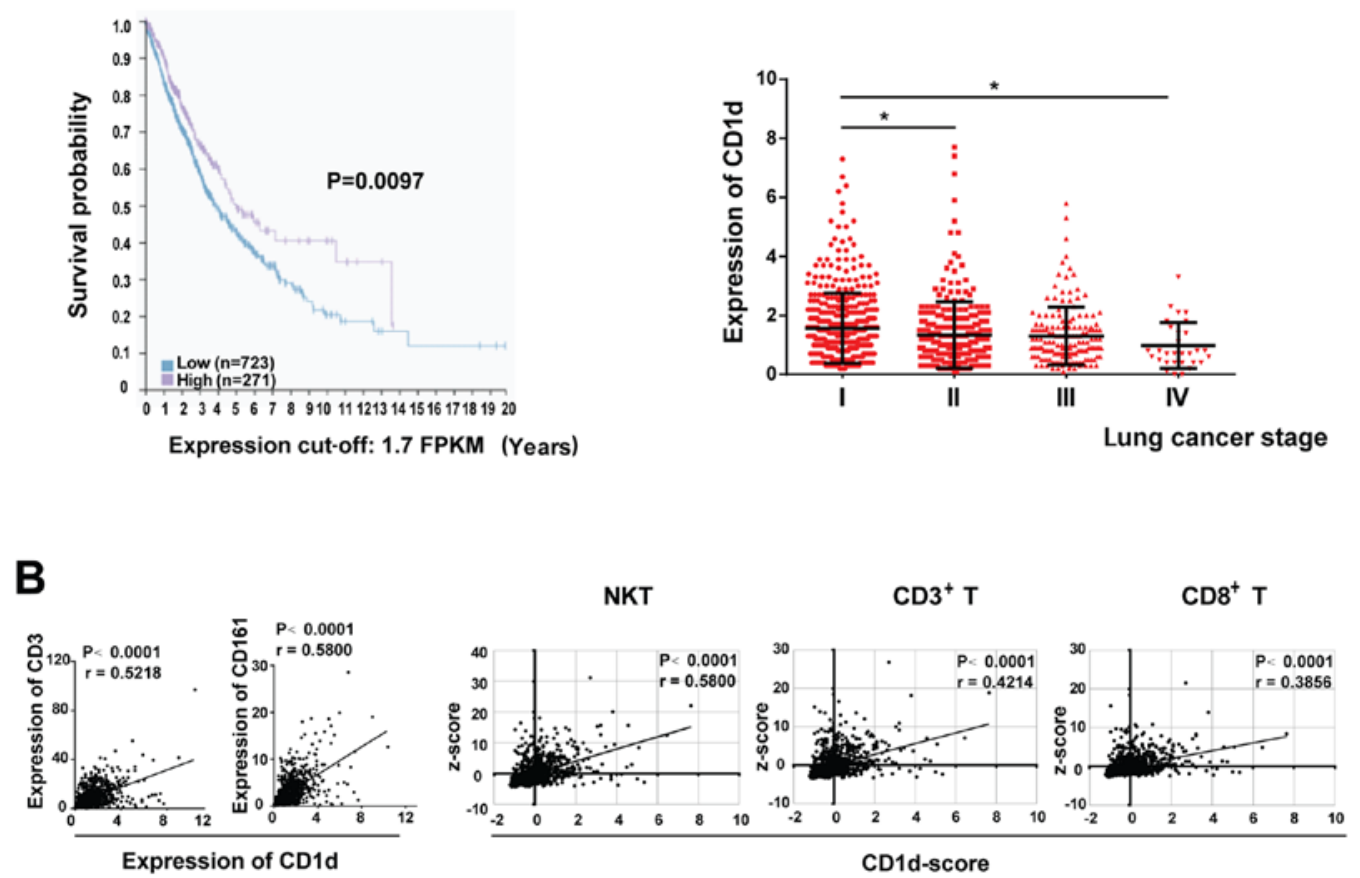

C
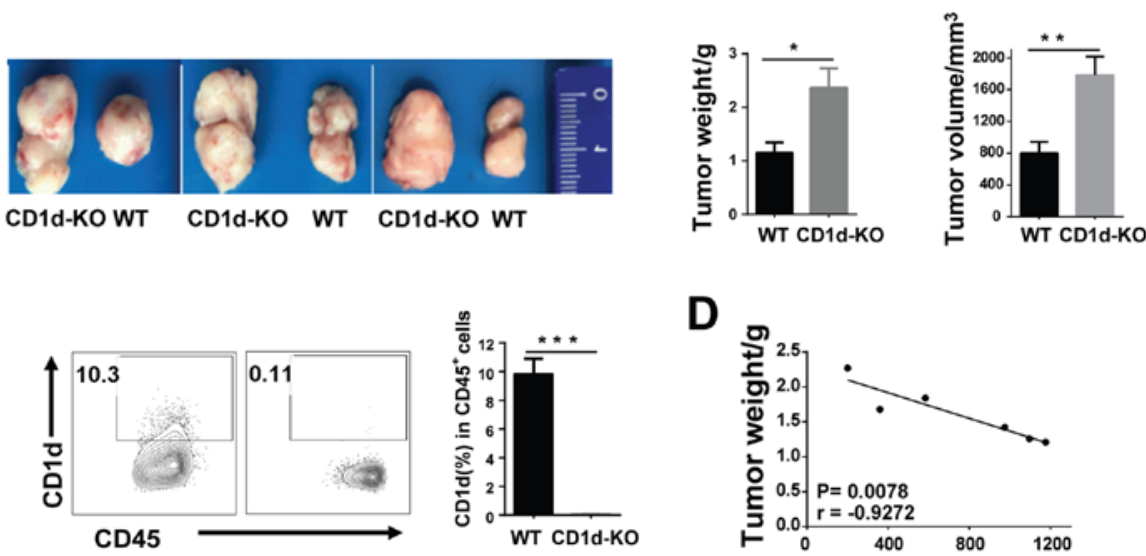

D

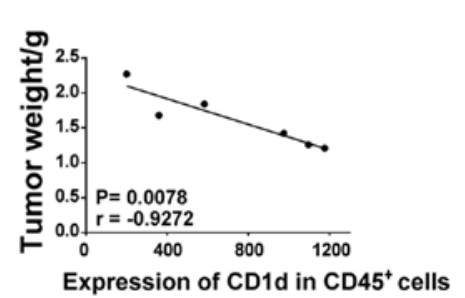

Figure 1. The expression of CD1d is correlated with the extended survival of lung cancer patients. (A) The Kaplan-Meier plots summarized the correlation between the mRNA expression level of CDld and lung cancer patient survival based on TCGA data. Patients were divided into one of two groups based on the level of CD1d; 'low ( $\mathrm{n}=723$ )' or 'high $(\mathrm{n}=271)$ ', cut-off was equal to 1.7FPKM. The TCGA data were derived from the Human Protein Atlas. CD1d expression was analyzed at different tumor stages ( $\mathrm{n}=994)$. (B) The correlation between CD1d and the expression levels of CD161 and CD3 in lung cancer patients. The NKT cell score was calculated by combining the expression levels of CD161, CD3, CD69, GZMB, CD107a and IFN- $\gamma$; the CD ${ }^{+} \mathrm{T}$ cell score was calculated by combining the expression levels of CD3, CD69, GZMB, CD107a and IFN- $\gamma$; and the CD8 ${ }^{+} \mathrm{T}$ cell score was calculated by combining the expression levels of CD8, CD69, GZMB and CD107a. Pearson's correlation coefficient was performed. (C) The tumor weight and volume in CD1d-KO and WT tumor-bearing mice. The MFI value of CD1d was detected by flow cytometry. (D) Correlation analysis between the MFI value of CD1d on CD45 ${ }^{+}$cells (spleen) and tumor weight. Data presented in $\mathrm{C}$ and $\mathrm{D}$ are pooled from three independent experiments, each with 3-4 mice and expressed as the mean $\pm \mathrm{SEM}$. ${ }^{*} \mathrm{P}<0.05,{ }^{* *} \mathrm{P}<0.01,{ }^{* * * *} \mathrm{P}<0.001$. NKT, natural killer T; MFI, median fluorescence intensity; WT, wild-type; KO, knockout; SEM, standard error of the mean; FPKM, fragments per kilobase million.

survival of patients, its level decreasing as the tumor stage progressed from I to IV (Fig. 1A). Moreover, the mRNA levels of markers of NKT cells (CD161), T cells (CD3), and even cytotoxic $\mathrm{T}$ cells $(C D 8)$ increased correspondingly with an increase in CD1d expression. By combining the expression levels of activation and antitumor-related genes [CD69, $C D 107 a, G Z M B$ (granzyme B) and $I F N-\gamma]$ of all the T cells, we found that high levels of CD1d may indicate a stronger antitumor activity (Fig. 1B).

To confirm the suppressive role of CDld in lung cancer, CD1d-deficent (CD1d-KO) mice were used. We established a 3LL-tumor-bearing model by subcutaneously inoculating 3LL cells into WT mice and CD1d-KO mice, respectively. We observed that the tumor weight and volume in CD1d-KO mice were significantly higher than those in WT mice, indicating that CD1d suppressed tumor growth (Fig. 1C). Consistently, in 3LL tumor-bearing WT mice, the weight of the tumor was inversely correlated with the median fluorescence intensity (MFI) value of CD1d (Fig. 1D). These results indicated that CD1d functioned as a tumor suppressor in lung cancer, which may be due to the enhancement of antitumor activities of NKT cells and T cells. 
A

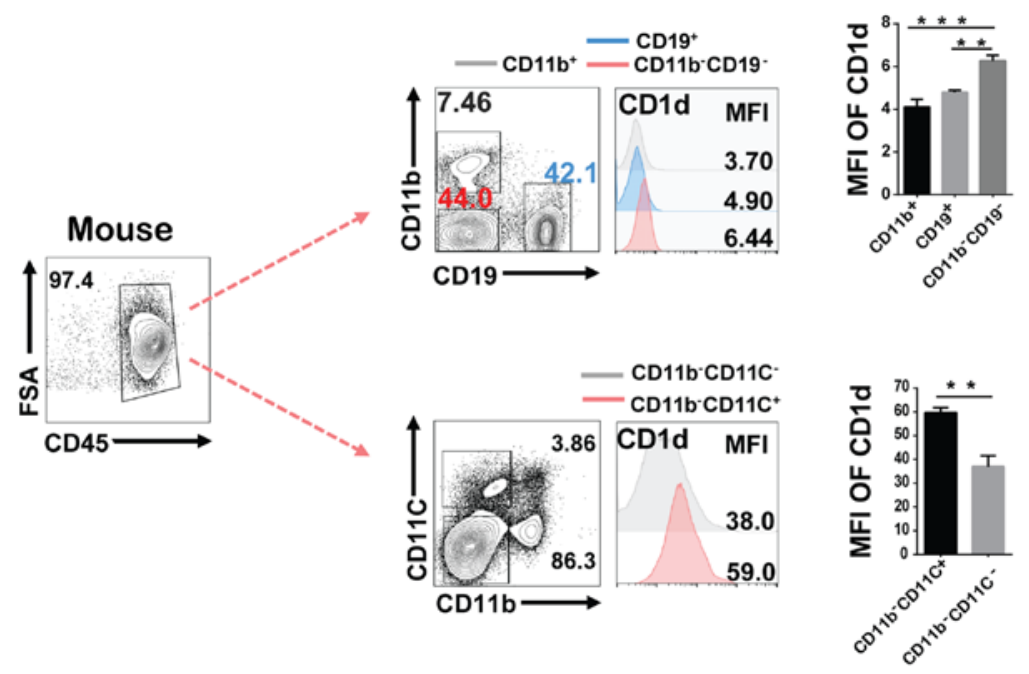

B

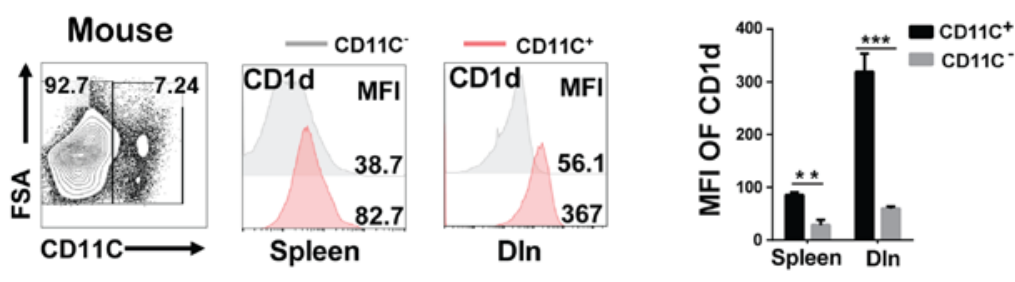

C

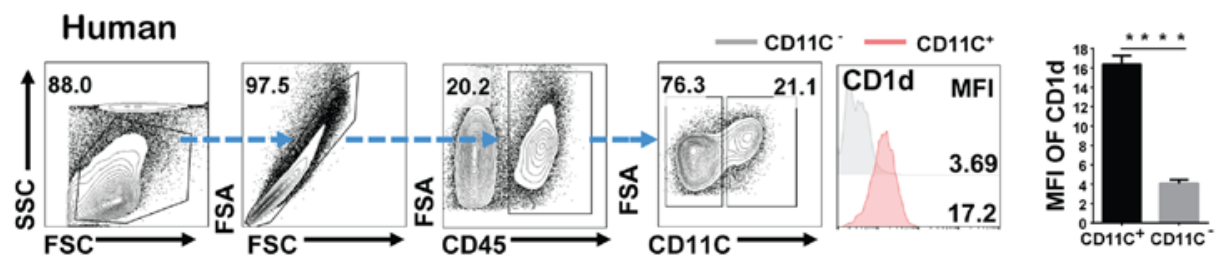

Figure 2. CD1d is mainly expressed on DCs of tumor-bearing mice. (A) The statistical summary for CD1d expression on the major antigen-presenting

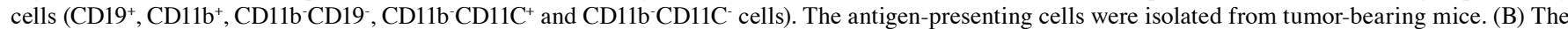

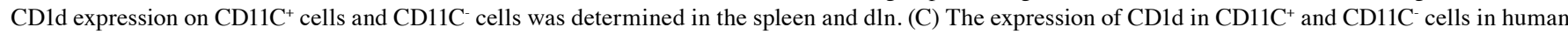
lung cancer tissues from lung cancer patients, $n=4$. Data presented in A and B are pooled from three independent experiments each with $3-4$ mice and expressed as the mean \pm SEM. ${ }^{* *} \mathrm{P}<0.01,{ }^{* * *} \mathrm{P}<0.001,{ }^{* * * *} \mathrm{P}<0.0001$. DCs, dendritic cells; dln, draining lymph nodes; MFI, median fluorescence intensity; SEM, standard error of the mean; FSA, FSC-A (area of FSC); SSC, SSC-H (height of SSC).

CDId is mainly expressed on DCs in the presence of tumors. CD1d is an important antigen-presenting molecule. To investigate whether the potential antitumor effect of CD1d was related to the stronger antigen presentation, we compared the CD1d expression on APCs in tumor-bearing mice by FACS. Results revealed that compared with $\mathrm{CD} 19^{+} \mathrm{B}$ cells and other $\mathrm{CD}_{11} \mathrm{~b}^{+}$monocytes-phagocytes, the CD11b-CD19-CD $11 \mathrm{C}^{+}$ DCs expressed the highest level of CD1d in tumor-bearing mice (Fig. 2A). Levels of CD1d on $\mathrm{CD}_{11 \mathrm{C}^{+}}$cells of the spleen and draining lymph node (dln) were both high when compared with the paired CD11C ${ }^{-}$cells (Fig. 2B). To confirm this result, we analyzed the CD1d expression on $\mathrm{CD} 11 \mathrm{C}^{+} \mathrm{DCs}$ and paired

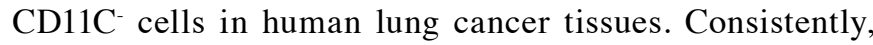
CD1d expression of DC cells was higher than the paired cells (Fig. 2C), which indicated that CD1d is mainly expressed on DCs in tumor-bearing mice.

Adoptive transfer of CDId DCs suppresses tumor growth, with increasing all the T cells activity. To further investigate whether the CD1d expression on DCs can enhance the antitumor effect of all the T cells, we isolated CD1d ${ }^{+}$and CD1d-KO DCs. They were then transferred respectively into CD11C-DTR mice injected with diphtheria toxin (DT), resulting in the 3LL tumor-bearing model being established (Fig. 3A). After

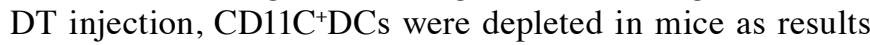
revealed (Fig. S1A). Subsequently, the isolated DCs were injected into the DC-deficient mice (Fig. S1B). After four weeks, the tumor weight and volume of CD1d ${ }^{+}$DC-transferred mice was significantly lower than those in mice with CD1d-KO DC (Fig. 3A). Moreover, we found that NKT cells $\left(\mathrm{CD}^{+}{ }^{+} \mathrm{NK} 1.1^{+}\right)$ and $\mathrm{T}$ cells $\left(\mathrm{CD}^{+} \mathrm{NK} 1.1^{-}\right)$were both markedly activated after transferring CD1d ${ }^{+}$DC into mice, with high levels of CD69 and CD107a, and more cytokines such as IFN- $\gamma$ (Fig. 3B). These results demonstrated that transferring $\mathrm{CD}^{-} \mathrm{d}^{+} \mathrm{DCs}$ can enhance activation of all the $\mathrm{T}$ cells and reduce tumor burden.

$C D 1 d^{+} D C s$ highly express MHCs and costimulatory molecules, exhibiting a stronger ability to present antigens to 


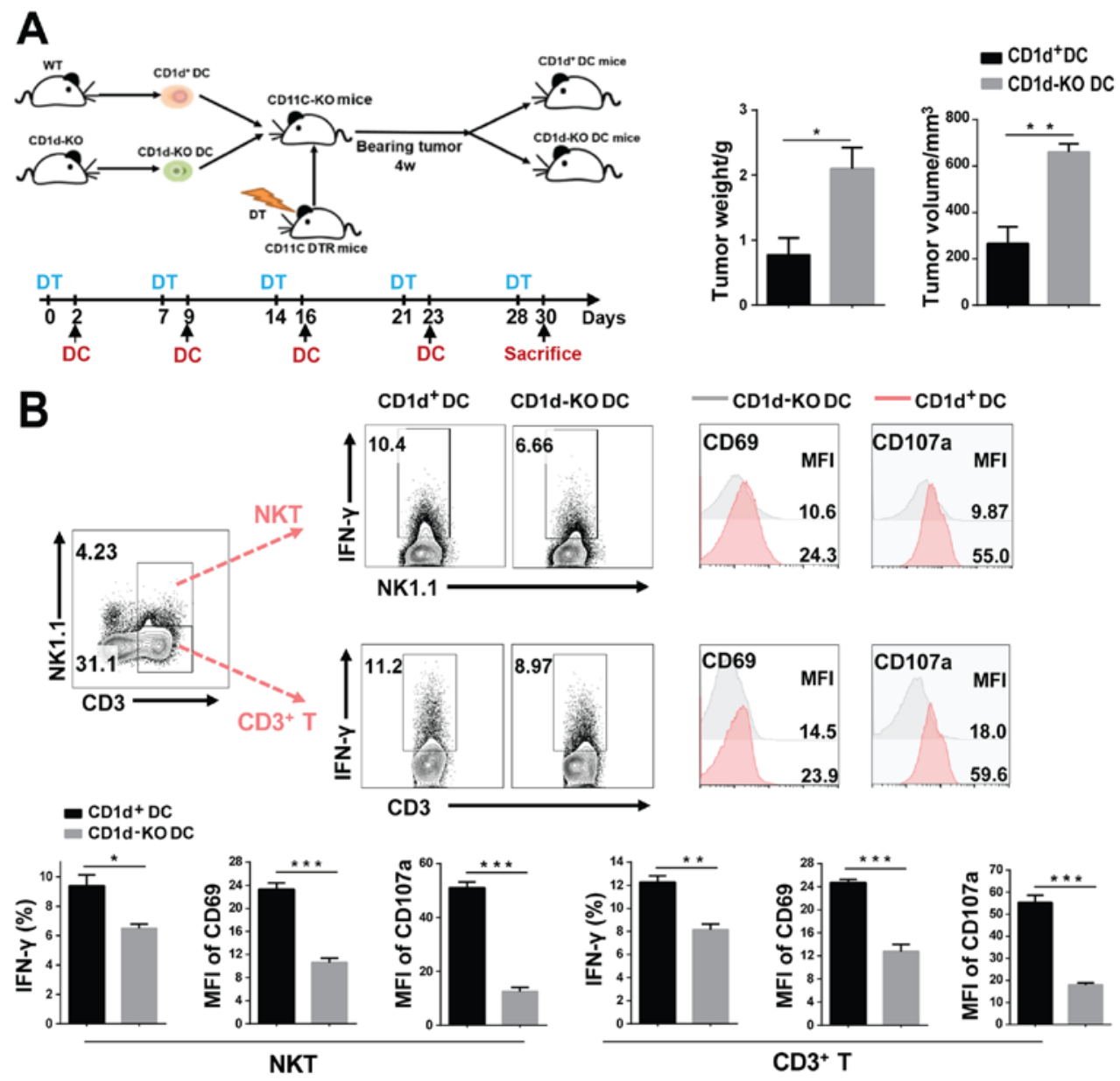

Figure 3. Adoptive transfer of CD1d ${ }^{+} \mathrm{DCs}$ suppresses tumor growth, with an increase in the activity of all T cells. (A) We transferred CD1d $\mathrm{d}^{+} \mathrm{Cs}$ and CD1d-KO DCs into CD11C-DTR mice which had been injected with DT previously, and then established the tumor-bearing model. (B) The weight and volume of the tumors are displayed in CD1d ${ }^{+} \mathrm{DC}$ - and CD1d-KO DC-transferred mice on day 28 of tumor transplantation. The expression of CD69 and CD107a, and IFN- $\gamma$ production of NKT and $\mathrm{CD}^{+} \mathrm{T}$ cells (from spleen) were detected by FACS. Data presented in A and B were pooled from three independent experiments each with three mice and expressed as the mean \pm SEM. ${ }^{*} \mathrm{P}<0.05,{ }^{* * *} \mathrm{P}<0.01,{ }^{* * *} \mathrm{P}<0.001$. DCs, dendritic cells; KO, knockout; DT, diphtheria toxin; FACS, fluorescence-activated cell sorter; SEM, standard error of the mean.

$T$ cells. Previous data have also shown that CD1d is essential for NKT cell activation, but little is known about its role in T-cell activation by DCs. In the present study, we confirmed that CD1d expression was required for DCs to present antigen to NKT cells, and subsequent NKT cell activation (Fig. S1C). FACS analysis revealed that the expression of CD107a and CD69, as well as the secretions of IFN- $\gamma$, were higher in NKT cells co-cultured with CD1d ${ }^{+}$DCs than those of NKT cells with CD1d-KO DCs (Fig. S1D).

To investigate whether the increased expression of CDld also affected the ability of DCs in presenting antigens to T cells, we examined the expression of antigen-presentation related MHC-I/II, as well as costimulatory molecules (e.g. CD80, CD86 and CD40) on CD1d ${ }^{+}$DCs and CD1d-DCs. The results revealed that higher levels of MHC-I/II, CD80, CD86 and CD40 were expressed by CD1d ${ }^{+}$DCs than by CD1d-DCs in 3LL tumor-bearing mice (Fig. 4A and S2A). Consistently, in human lung cancer tissues, levels of HLA-A/B/C and costimulatory molecules for activating $\mathrm{T}$ cells were also consistently higher in CD1d ${ }^{+}$DCs than in their paired CD1d-DCs (Fig. 4B and S2B). These results were confirmed by TCGA data, which revealed that the CD1d ${ }^{+} \mathrm{DC}$ score (combining CDId and $C D 11 C$ ) was positively correlated with human MHC-I/II, and costimulatory molecules for activating $\mathrm{T}$ cells (Fig. 5A). Moreover, signals such as NF- $\mathrm{kB}$ (p65) and Erk1/2 that were reported as essential for the expression of MHCs and costimulatory molecules were also markedly activated. In addition, JAK2-STAT3/6 signaling was involved in maintaining the high level of CD1d on DCs (Fig. 5B). These results indicated that CD1d expression on DCs was not only required for NKT cell activation, but also endowed DCs with a strong capacity to present antigens to T cells (Figs. 4 and 5B).

Antitumor activities of NKT, $C D 4^{+} T$ and $C D 8^{+} T$ cells are enhanced in lung cancer patients with more CDId+DCs. Next, we confirmed our results in lung cancer patients. Six fresh lung cancer tissues were collected, in which, the percentage of CD1d ${ }^{+}$DCs and activation of all the $\mathrm{T}$ cells were detected by FACS. Subsequently, the correlation of CD1d ${ }^{+} \mathrm{DC}$ percentage with activation in all the $\mathrm{T}$ cells was analyzed. The results revealed that patients with more $C D 1 d^{+} D C$ cells had stronger antitumor immunity, particularly $\mathrm{T}$ cell-related antitumor response. With an increase in levels of CD1d+DC cells in lung cancer tissues, $\mathrm{CD}^{+} \mathrm{T}$ cells also expressed a higher level of 
A

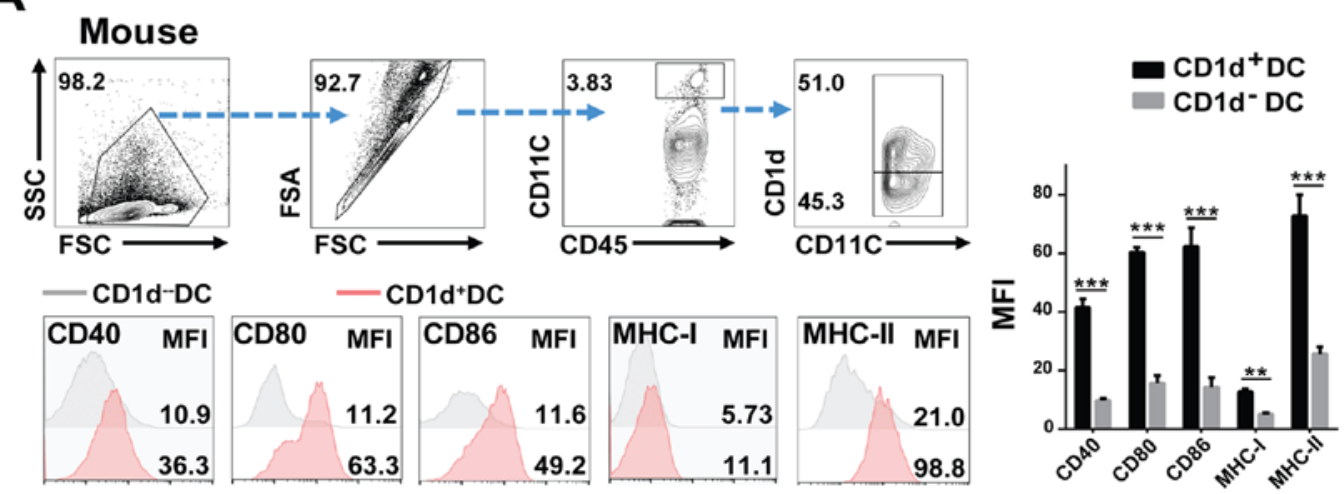

B

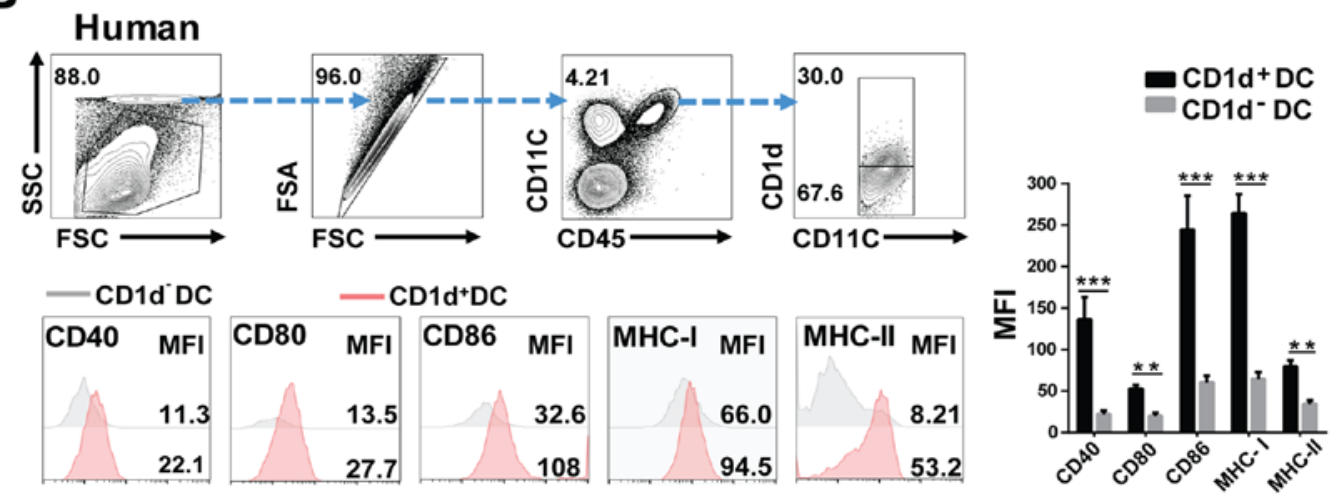

Figure 4. CDld ${ }^{+} \mathrm{DCs}$ highly express MHCs and costimulatory molecules, revealing a stronger ability to present antigens to $\mathrm{T}$ cells. (A) The gating strategies of flow cytometric analysis and flow cytometric analysis of CD40, CD80, CD86, MHC-I/II expression on splenic CD1d ${ }^{+}$DCs and CD1d-DCs. The splenic cells were isolated from the tumor-bearing model (day 28). (B) Flow cytometric analysis of CD40, CD80, CD86 and MHC-I/II expression levels in human lung cancer tissues from lung cancer patients, $n=4$. Data presented in A and B were pooled from three independent experiments each with 3-4 mice and expressed as the mean \pm SEM. ${ }^{* * *} \mathrm{P}<0.01,{ }^{* * * *} \mathrm{P}<0.001$. DCs, dendritic cells; MFI, median fluorescence intensity; SEM, standard error of the mean; FSA, FSC-A (area of FSC); SSC, SSC-H (height of SSC).

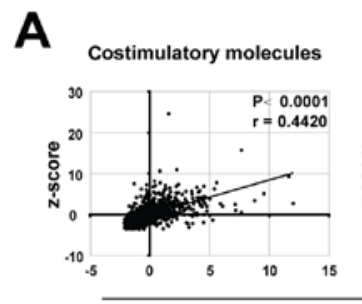

B
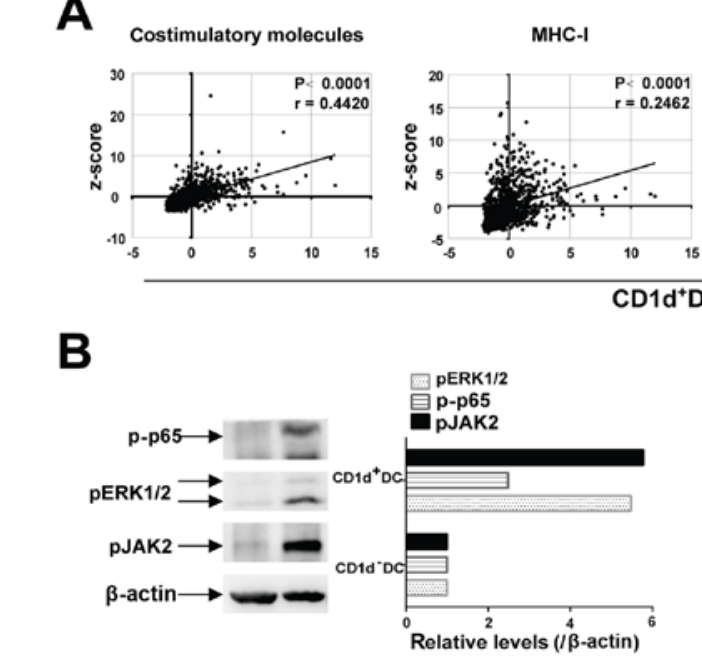
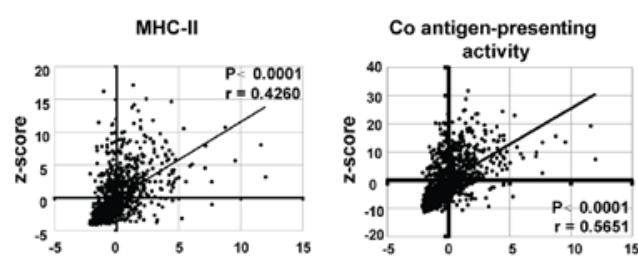

CD1d + DC score (human)
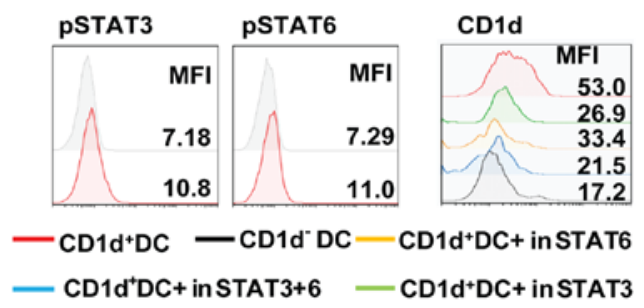

Figure 5. (A) The correlation analysis between $\mathrm{CD} 1 \mathrm{~d}^{+} \mathrm{DC}$ cell score and Co antigen-presenting activity score. The costimulatory molecule scores were calculated by combining the expression levels of CD40, CD80 and CD86; the MHC-I score was calculated by combining the expression levels of HLA-A/B/C; and the MHC-II score was calculated by combining the expression levels of HLA-DMA/DOA/DQA1/DRA. The co antigen-presenting activity score was calculated by combining the expression levels of CD40, CD80, CD86 and MHC-I/II. Pearson's correlation coefficient was performed. (B) Protein levels of pp65, pERK1/2 and pJAK2 in CD1d ${ }^{+}$DC cells and CD1d-DC cells were detected via western blot analysis. FACS analysis of pSTAT6 and pSTAT3 in CD1d ${ }^{+} D C$ cells and CD1d-DC cells. CD1d ${ }^{+}$DC cells were treated with inhibitors of pSTAT3 $(10 \mathrm{nM})$ and pSTAT6 $(10 \mathrm{nM})$, and then expression levels of CD1d were detected by FACS. DC, dendritic cells; MFI, median fluorescence intensity; FACS, fluorescence-activated cell sorter.

activation-related CD69, cytotoxic effect-associated CD107a, and GZMB (Fig. 6B) in addition to NKT cells (Fig. 6A).
Among $\mathrm{CD}^{+} \mathrm{T}$ cells, the antitumor activity of cytotoxic $\mathrm{CD}^{+} \mathrm{T}$ cells significantly increased with upregulation of 


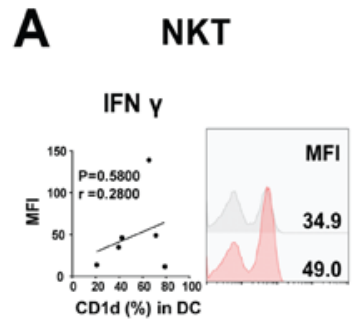

CD69

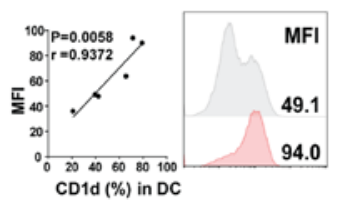

GZMB

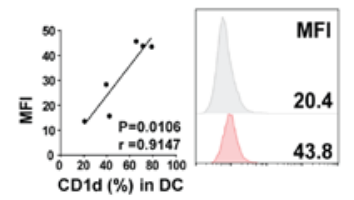

CD107a

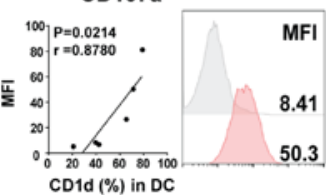

B $\quad \mathrm{CD}^{+} \mathrm{T}$

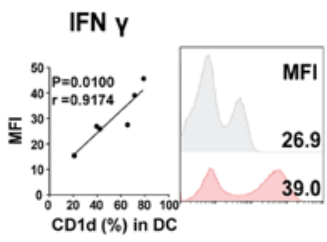

CD69

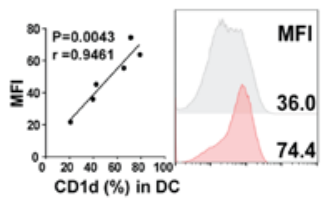

GZMB

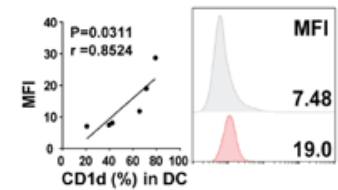

CD107a

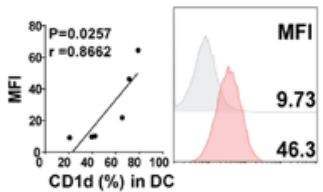

C $\quad \mathrm{CD}^{+} \mathrm{T}$
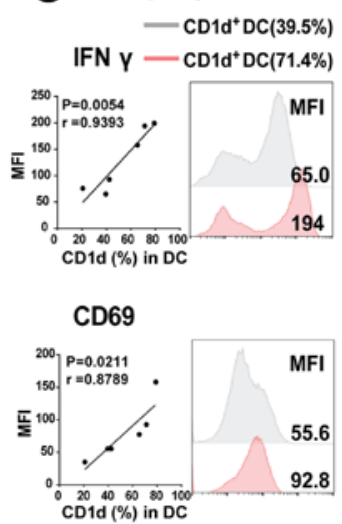

GZMB
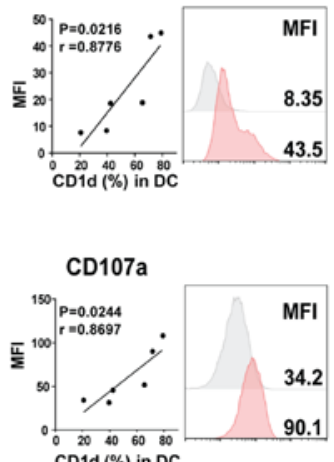

D

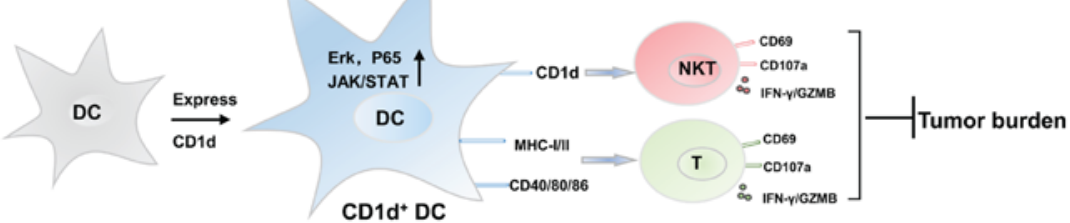

Figure 6. Antitumor activities of all the T cells are enhanced in lung cancer patients with more CD1d ${ }^{+}$DCs. (A-C) Flow cytometric analysis of IFN- $\gamma$, CD69, GZMB and CD107a, expression on NKT, CD3 ${ }^{+} \mathrm{T}$ and $\mathrm{CD} 8^{+} \mathrm{T}$ cells in human lung cancer tissues from lung cancer patients, $\mathrm{n}=6$. (D) A proposed model for all the results. All values are presented as the mean \pm SEM. DCs, dendritic cells; MFI, median fluorescence intensity; GZMB, granzyme B; NKT, natural killer T; SEM, standard error of the mean.

CD1d+DC cells (Fig. 6C). These data confirmed that increasing CD1d ${ }^{+} \mathrm{DC}$ cell levels in patients also promoted the activation of $\mathrm{T}$ cells, particularly cytotoxic $\mathrm{CD} 8^{+} \mathrm{T}$ cells, thus enhancing antitumor immunity

\section{Discussion}

CD1d, an MHC-I like molecule can present phospholipid and glycosphingolipid antigens (Ags) to natural killer T (NKT) cells, which regulate nearly all aspects of both the innate and adaptive immune responses. However, it is uncertain whether CDld-positive dendritic cells (DCs) may produce an antitumor effect by activating $T$ cells. The present study revealed that CD1d was predominantly expressed on DCs, which suppressed tumor growth. CDld ${ }^{+} \mathrm{DCs}$ highly expressed MHCs and co-stimulatory molecules, exhibiting a stronger ability to activate $\mathrm{T}$ cells. The adoptive transfer of CD1d ${ }^{+}$DCs improved the antitumor effect of all the T cells, thus suppressing tumor growth. Lung cancer patients with higher levels of CDld ${ }^{+} \mathrm{DCs}$ also exhibited a greater activated antitumor response of $\mathrm{T}$ cells, particularly cytotoxic
T cells. Collectively, our studies revealed the importance of CD1d on DCs for enhancing antitumor immunity in lung cancer. Additionally, we also analyzed the CD1d expression in other types of tumors based on TCGA data, and found that in other tumors such as endometrial and pancreatic cancer, levels of CD1d were also positively correlated with the overall survival of patients, implying that the potential antitumor ability of CDld ${ }^{+}$DC may be not specific for lung cancer (Fig. S3A).

CD1d is an important antigen-presentation molecule. The tumor antigen contains types of lipid and glycolipid antigens, such as $N$-glycolyl-monosialodihexosyl-ganglioside (NGcGM3) (23), T antigen (a disaccharide hidden on normal cells, but can be selectively exposed on the surface of a diverse range of tumor cells) (24). Thus, the polarization/development of CD1d ${ }^{+} \mathrm{DCs}$ may be related to these antigens in tumor cells. Although, there is no direct data revealing which key factors regulate the function of $\mathrm{CD} 1 \mathrm{~d}^{+} \mathrm{DCs}$, we demonstrated that CD1d ${ }^{+}$DCs highly expressed antigen-presentation associated molecules (e.g. MHC-I/II, CD40, CD80 and CD86), implying their stronger ability of antigen-presentation to all the $\mathrm{T}$ cells. 
Furthermore, when DCs upregulate surface markers such as CD80, CD83 and CD86 along with the MHC-I/II, phenotypic maturation is gradually attained (25). Therefore, CD1d ${ }^{+} \mathrm{DCs}$ highly expressing MHCs and costimulatory molecules (CD40, CD80 and CD86) are mainly similar to mature DCs.

Compared with the non-tumor control group, CD1d expression on DCs in tumor-bearing mice and human lung cancer tissues was upregulated (Fig. S3B), which enhanced the antitumor effect of DCs. However, TCGA data revealed that, as the tumor progressed, the CD1d expression in tumor tissues was downregulated (Fig. 1A), implying that CD1d ${ }^{+} \mathrm{DCs}$ may be impaired in the local tumor microenvironment (TME), resulting in failure to resolve the tumor. Evident now is the fact that tumor-infiltrating DCs are often impaired, which leads these DCs to confer immune suppression (26). For instance, Liu et al reported that murine lung tumor cells released large amounts of PGE2 and TGF $\beta$ which resulted in the conversion of immune-activating DCs into immune-suppressive DCs

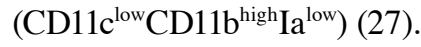

In the present study, we discovered that CD1d suppressed tumor growth, mainly since CD1d-positive DCs could enhance the antitumor effect of all the T cells. Conversely, a previous study reported that compared to WT mice, CD1d-KO mice had markedly fewer and smaller colon tumors in colitis-associated colon cancer (CRC) $(13,28)$. In this process, CD1d on intestinal epithelial cells may recruit neutrophils and cause more severe clinical adjacent normal colitis $(13,29)$. Considering the contrary, we speculated that this was due to the alleviation of colitis in CD1d-KO mice which resulted in a decrease in tumor growth. However, in the 3LL-bearing tumor model, tumorigenesis is not predominantly driven by inflammation. In agreement with our data, the antitumor effect of CD1d has been reported in several studies using experimental models such as brain tumors and prostate carcinomas (30). For instance, CD1d-positive medulloblastoma (MB) cells effectively cross-present glycolipid antigens and can be killed by NKT cells both in vitro and in vivo (31).

CD1d expressed on other APCs may mediate different responses. For instance, B cells with CD1d expression are considered a mechanism of immune evasion, and have both diagnostic and prognostic importance (32). A splenic CD5 ${ }^{+} \mathrm{CD} 1 \mathrm{~d}^{\text {hi }}$ subset was identified as important regulatory B cells (Bregs), which suppressed ongoing immune responses by secreting IL-10. In addition, CD1d-lipid presentation by Bregs induced NKT cells to secrete IFN- $\gamma$ to contribute to the downregulation of T helper (Th1) and Th17-adaptive immune responses and ameliorated experimental arthritis. In addition, CD1d expression has been demonstrated in human cancers, where it plays a diverse role in different tumors (30). For instance, CD1d-positive gliomas are susceptible to ex vivo activated NKT cell killings (12), whereas the expression of CD1d in renal cell carcinoma (RCC) was associated with aggressive disease and poorer clinical outcomes (28). Collectively, the expression of CD1d on cells is suggestive of the ability of these cells to present Ag to, and form cognate interactions with, NKT cells. Although current experimental evidence suggests an important role for CD1d and NKT cells in health and disease, further studies are required to better define their role in this context (11).

In addition to targeting NKT cells, mature DCs also have the ability to present pathogen-derived antigens to specific $\mathrm{T}$ cells Notably, the data presented in this study revealed that CD1d overexpression could strengthen this ability. The enhancement of their potent antigen-presenting strategies may be sufficient to strongly activate low-affinity effector T cells and break the natural tolerance towards endogenous tumor-associated antigens (TAAs). DCs present on their surface a wide collection of receptors which recognize a variety of pathogen-associated molecular patterns, or inflammation-related cellular proteins. The binding of these molecules with their appropriate receptors triggers the phenotypic and functional maturation of DCs, through the strong upregulation of $\mathrm{MHC}$ and costimulatory molecules of antigen presentation (33). For example, PD-L1 delivers an inhibitory signal to T cells by binding to PD-1 (34). However, it is reported that PD-L1 silencing in antigen-presenting DCs interfered with ligand-induced TCR down-modulation in $\mathrm{CD}^{+} \mathrm{T}$ cells. Additionally, tumor-derived cytokine thymic stromal lymphopoietin (TSLP) upregulated the expression of OX40L on DCs, thereby instructing them to generate $\mathrm{CD}^{+}{ }^{+} \mathrm{Th} 2$ cells that produce the tumor-supporting cytokines IL-4 and IL-13. In the present study, our results revealed that the overexpression of CD1d on DCs enhanced T cell-mediated antitumor effects (35). The adoptive transfer of CD1d-positive DCs into tumor-bearing mice resulted in an effective immunotherapy, indicating a potential for clinical application to cancer patients.

As aforementioned, DC vaccination may be one of the best immunotherapeutic strategies (36). Several strategies have been used to boost DC antigen-presenting functions, but cancer immunotherapy is not as effective as anticipated according to preclinical models. In this study, we propose that CD1d ${ }^{+} \mathrm{DCs}$ that have a stronger capacity to induce the antitumor effects of NKT, CD4 ${ }^{+} \mathrm{T}$ and $\mathrm{CD}^{+} \mathrm{T}$ cells, may be superior to the bulk population of DCs in cancer immunotherapy.

\section{Acknowledgements}

The CD11C-DTR mice were provided by Professor Zhinan Yin (College of Life Sciences, Nankai University, Tianjin, China).

\section{Funding}

The present study was supported by the National Science Foundation of China (grant no. 81601362), and the Shanghai Municipal Commission of Health and Family Planning (grant nos. 2018YQ16 and 20184Y0089).

\section{Availability of data and materials}

The datasets generated and/or analyzed during the present study are available in the [Human Protein Atlas] repository, [http://www.proteinatlas.org/].

\section{Authors' contributions}

YL and CZ completed all trial procedures, data analysis and were major contributors in writing the manuscript. RL and JG designed the overall idea of the experiment and provided theoretical guidance throughout the process, participated in the processing of data, as well as prepared the final version of the manuscript. JL, ZL performed the cell biological experiments. ML revised the manuscript and contributed to the design of the study. All authors read and approved the manuscript 
and agree to be accountable for all aspects of the research in ensuring that the accuracy or integrity of any part of the work are appropriately investigated and resolved.

\section{Ethics approval and consent to participate}

The study was approved by the Zhongshan Hospital Research Ethics Committee. Informed consents were obtained from all patients.

\section{Patient consent for publication}

Not applicable.

\section{Competing interests}

The authors declare that they have no competing interests.

\section{References}

1. Escors D: Tumour immunogenicity, antigen presentation and immunological barriers in cancer immunotherapy. New J Sci 2014 pii: $734515,2014$.

2. Bandola-Simon J and RochePA:Dysfunction of antigen processing and presentation by dendritic cells in cancer. Mol Immunol Apr 5, 2018 (Epub ahead of print). pii: S0161-5890(18)30104-4, doi: 10.1016/j.molimm.2018.03.025.

3. Cordes LM, Gulley JL and Madan RA: Perspectives on the clinical development of immunotherapy in prostate cancer. Asian J Androl 20: 253-259, 2018.

4. Chen YL, Chang MC, Chiang YC, Lin HW, Sun NY, Chen CA, Sun WZ and Cheng WF: Immuno-modulators enhance antigenspecific immunity and anti-tumor effects of mesothelin-specific chimeric DNA vaccine through promoting DC maturation. Cancer Lett 425: 152-163, 2018.

5. de Charette M, Marabelle A and Houot R: Turning tumour cells into antigen presenting cells: The next step to improve cancer immunotherapy? Eur J Cancer 68: 134-147, 2016.

6. Jung NC, Lee JH, Chung KH, Kwak YS and Lim DS: Dendritic cell-based immunotherapy for solid tumors. Transl Oncol 11: 686-690, 2018

7. Canchis PW, Bhan AK, Landau SB, Yang L, Balk SP and Blumberg RS: Tissue distribution of the non-polymorphic major histocompatibility complex class I-like molecule, CD1d. Immunology 80: 561-565, 1993.

8. Barral DC and Brenner MB: CD1 antigen presentation: How it works. Nat Rev Immunol 7: 929-941, 2007.

9. Moody DB and Porcelli SA: Intracellular pathways of CD1 antigen presentation. Nat Rev Immunol 3: 11-22, 2003.

10. McEwen-Smith RM, Salio M and Cerundolo V: CD1d-dependent endogenous and exogenous lipid antigen presentation. Curr Opin Immunol 34: 116-125, 2015.

11. Brutkiewicz RR, Yunes-Medina L and Liu J: Immune evasion of the CD1d/NKT cell axis. Curr Opin Immunol 52: 87-92, 2018.

12. Teo WY, Elghetany MT, Shen J, Man TK, Li X, Chintagumpala M, Su JM, Dauser R, Whitehead W, Adesina AM, et al: Therapeutic implications of CD1d expression and tumor-infiltrating macrophages in pediatric medulloblastomas. J Neurooncol 120 293-301, 2014

13. Huang E, Liu R, Lu Z, Liu J, Liu X, Zhang D and Chu Y: NKT cells mediate the recruitment of neutrophils by stimulating epithelial chemokine secretion during colitis. Biochem Biophys Res Commun 474: 252-258, 2016.

14. Webb TJ, Carey GB, East JE, Sun W, Bollino DR, Kimball AS and Brutkiewicz RR: Alterations in cellular metabolism modulate CD1d-mediated NKT-cell responses. Pathog Dis 74: 74, 2016.

15. Chung BK, Priatel JJ and Tan R: CDld Expression and invariant NKT cell responses in herpesvirus infections. Front Immunol 6: $312,2015$.

16. Exley M, Garcia J, Wilson SB, Spada F, Gerdes D, Tahir SM, Patton KT, Blumberg RS, Porcelli S, Chott A, et al: CD1d structure and regulation on human thymocytes, peripheral blood $\mathrm{T}$ cells, B cells and monocytes. Immunology 100: 37-47, 2000.
17. Brigl M and Brenner MB: CD1: Antigen presentation and T cell function. Annu Rev Immunol 22: 817-890, 2004.

18. Männ L, Kochupurakkal N, Martin C, Verjans E, Klingberg A, Sody S, Kraus A, Dalimot J, Bergmüller E, Jung S, et al: CD11c. DTR mice develop a fatal fulminant myocarditis after local or systemic treatment with diphtheria toxin. Eur J Immunol 46: 2028-2042, 2016.

19. Wang SS, Liu W, Ly D, Xu H, Qu Land Zhang L: Tumor-infiltrating B cells: Their role and application in anti-tumor immunity in lung cancer. Cell Mol Immunol, 2018.

20. Zhou J, Lai W, Yang W, Pan J, Shen H, Cai Y, Yang C, Ma N, Zhang Y,Zhang R, et al: BLT1 in dendritic cells promotes Th1/Th17 differentiation and its deficiency ameliorates TNBS-induced colitis. Cell Mol Immunol 15: 1047-1056, 2018.

21. Zheng Y, Xiong S, Jiang P, Liu R, Liu X, Qian J, Zheng X and Chu Y: Glucocorticoids inhibit lipopolysaccharide-mediated inflammatory response by downregulating microRNA-155: A novel anti-inflammation mechanism. Free Radic Biol Med 52: 1307-1317, 2012.

22. Perwitasari O, Cho H, Diamond MS and Gale M Jr: Inhibitor of $\kappa \mathrm{B}$ kinase epsilon (IKK(epsilon)), STAT1, and IFIT2 proteins define novel innate immune effector pathway against West Nile virus infection. J Biol Chem 286: 44412-44423, 2011.

23. Gentilini MV, Pérez ME, Fernández PM, Fainboim L and Arana E: The tumor antigen $N$-glycolyl-GM3 is a human CD1d ligand capable of mediating $\mathrm{B}$ cell and natural killer $\mathrm{T}$ cell interaction. Cancer Immunol Immunother 65: 551-562, 2016.

24. Ferguson K, Yadav A, Morey S, Abdullah J, Hrysenko G, Eng JY, Sajjad M, Koury S and Rittenhouse-Olson K: Preclinical studies with JAA-F11 anti-Thomsen-Friedenreich monoclonal antibody for human breast cancer. Future Oncol 10: 385-399, 2014.

25. Reis e Sousa C: Dendritic cells in a mature age. Nat Rev Immunol 6: 476-483, 2006.

26. Veglia F and Gabrilovich DI: Dendritic cells in cancer: The role revisited. Curr Opin Immunol 45: 43-51, 2017.

27. Liu Q, Zhang C, Sun A, Zheng Y, Wang L and Cao X: Tumoreducated CD11b $b^{\text {high }} a^{\text {low }}$ regulatory dendritic cells suppress $\mathrm{T}$ cell response through arginase I. J Immunol 182: 6207-6216, 2009.

28. Chong TW, Goh FY, Sim MY, Huang HH, Thike AA, Lim WK, Teh BT and Tan PH: CD1d expression in renal cell carcinoma is associated with higher relapse rates, poorer cancer-specific and overall survival. J Clin Pathol 68: 200-205, 2015.

29. Sáez de Guinoa J, Jimeno R, Gaya M, Kipling D, Garzón MJ, Dunn-Walters D, Ubeda C and Barral P: CDld-mediated lipid presentation by $\mathrm{CD} 11 \mathrm{c}^{+}$cells regulates intestinal homeostasis. EMBO J 37: 37, 2018.

30. Hix LM, Shi YH, Brutkiewicz RR, Stein PL, Wang CR and Zhang M: CD1d-expressing breast cancer cells modulate NKT cell-mediated antitumor immunity in a murine model of breast cancer metastasis. PLoS One 6: e20702, 2011.

31. Liu D, Song L, Brawley VS, Robison N, Wei J, Gao X, Tian G, Margol A, Ahmed N, Asgharzadeh S, et al: Medulloblastoma expresses CD1d and can be targeted for immunotherapy with NKT cells. Clin Immunol 149: 55-64, 2013.

32. Oleinika K, Rosser EC, Matei DE, Nistala K, Bosma A, Drozdov I and Mauri C: CD1d-dependent immune suppression mediated by regulatory B cells through modulations of iNKT cells. Nat Commun 9: 684, 2018.

33. Liechtenstein T, Dufait I, Lanna A, Breckpot K and Escors D: Modulating co-stimulation during antigen presentation to enhance cancer immunotherapy. Immunol Endocr Metab Agents Med Chem 12: 224-235, 2012.

34. Karwacz K, Bricogne C, MacDonald D, Arce F, Bennett CL, Collins $\mathrm{M}$ and Escors D: PD-L1 co-stimulation contributes to ligand-induced $\mathrm{T}$ cell receptor down-modulation on $\mathrm{CD} 8^{+} \mathrm{T}$ cells. EMBO Mol Med 3: 581-592, 2011.

35. Liechtenstein T, Dufait I, Bricogne C, Lanna A, Pen J, Breckpot K and Escors D: PD-L1/PD-1 co-stimulation, a brake for T cell activation and a T cell differentiation signal. J Clin Cell Immunol S12: pii: 006, 2012.

36. Merad M, Sathe P, Helft J, Miller J and Mortha A: The dendritic cell lineage: Ontogeny and function of dendritic cells and their subsets in the steady state and the inflamed setting. Annu Rev Immunol 31: 563-604, 2013.

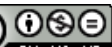

This work is licensed under a Creative Commons Attribution-NonCommercial-NoDerivatives 4.0 International (CC BY-NC-ND 4.0) License. 